\title{
Migración forzada de clases a formato online: un estudio de caso en la formación del profesorado de Música en el contexto COVID-19
}

\section{Forced migration to online classes: a case study in Music teacher education in the context of COVID-19}

Pedro Iglesias Calonge pedro.iglesias@umayor.c

Escuela Educación

Universidad Mayor

Santiago de Chile, Chile

ORCID: https://orcid.org/0000-0003-4678-3365

Lorena Rivera Pino

lorena.rivera@umayor.cl

Escuela Educación

Universidad Mayor

Santiago de Chile, Chile

ORCID: https://orcid.org/0000-0002-8853-444X

doi: 10.7203/LEEME.48.21700

Recibido: 30-09-2021 Aceptado: 26-11-2021. Contacto y correspondencia: Pedro Iglesias Calonge, Escuela Educación Universidad Mayor, Av. Manuel Montt 318, C.P. 7500628, Santiago de Chile. Chile

Resumen

Como medida de emergencia en respuesta a la pandemia producida por el COVID-19, la mayoría de las universidades en el mundo debió cerrar sus campus físicos y reprogramar sus actividades en formato online. Si bien las estadísticas oficiales indican que prácticamente la totalidad de las instituciones continuó con sus actividades formativas, la responsabilidad de las modificaciones didácticas y curriculares recayó sobre el profesorado que venía realizando clase en formatos presenciales y que no tenía experiencia en realizar docencia en formato online. Esta migración forzada significó que las universidades pudieron continuar con la formación del estudiantado de forma más o menos regular. No obstante, la estadística oficial que habla de tasas de aprobación no da cuenta de la efectividad del aprendizaje en este nuevo formato. A través una combinación de diseño de investigación de teoría fundamentada y un estudio de caso en una carrera de Pedagogía en Música en una universidad privada chilena, se busca describir, argumentar y evaluar desde el punto de vista del profesorado las modificaciones realizadas a las diferentes asignaturas, visualizar las dificultades que tuvieron durante el período y analizar sus percepciones sobre la efectividad y proyección de la enseñanza online en disciplinas musicales. Nuestros resultados apuntan a una gran diversidad de modificaciones realizadas por cada docente, destacando varias relacionadas con las metodologías activas, como el Aprendizaje Basado en Proyectos y el Aula Invertida. También, se da cuenta de una sobrecarga laboral importante y una percepción de que los aprendizajes se ven disminuidos en contextos online respecto a los presenciales.

Palabras clave: Formación inicial docente; enseñanza online; COVID-19; profesorado de Música.

\section{Abstract}

As an emergency measure in response to the pandemic produced by COVID-19, most of the universities in the world had to close their campuses and reschedule their activities. Although official statistics indicate that practically all the institutions continued with their learning activities, responsibility for didactic and curricular modifications fell on the teachers who had been giving classes in face-to-face formats and who had no experience in teaching in online formats. This forced migration meant that the universities were able to continue training students on a more or less regular basis; however, the official statistics that speak of approval rates do not account for the effectiveness of learning in this new format. The combination of a case study in a career in music pedagogy at a PLACE and an investigation of grounded theory design seeks to describe, substantiate, and evaluate the modifications made to the different subjects from the teacher's point of view, visualize the difficulties they had during the period, and reflect on the effectiveness and projection of online teaching in musical disciplines. Our results point to a great diversity of modifications, highlighting several related to Active Methodologies, such as Project based Learning and the Flipped Classroom. They also report a feeling of significant work overload and a perception that learning is diminished in online contexts compared to face-to-face ones.

Key words: Teacher Education; Online Learning; COVID-19; Music Teachers. 


\section{Introducción}

Durante 2020, la mayoría de los campus universitarios del mundo debieron cerrar como respuesta a la propagación del virus del COVID-19 (OECD, 2021). Chile no fue la excepción, de manera que a partir de fines de marzo del 2020 la carrera de Pedagogía en Artes Musicales de una universidad privada ubicada en Santiago de Chile, debió cerrar todos sus campus y suspender las actividades programadas en un semestre que recién comenzaba. Esto significó, al igual que en gran parte del mundo, modificar las actividades curriculares que estaban programadas de forma presencial y transformarlas a un formato online (Marinoni et al., 2020; OECD, 2021; UNESCO, 2021). No está documentado de manera global cuáles fueron las modificaciones ni quiénes fueron los responsables de las readecuaciones, ni mucho menos si se cumplieron los objetivos planificados originalmente.

En el contexto del caso estudiado, si bien la universidad se preocupó de proveer plataformas virtuales para realizar la docencia de manera remota, la responsabilidad de modificaciones curriculares y actividades cayó sobre el profesorado responsable de cada asignatura. Estos, en la mayoría de los casos, tenían poca o nula experiencia realizando docencia en modalidad online. Al igual que en todo el mundo, fue sorprendente verificar lo poco preparadas que estaban las instituciones de educación superior para este cambio (OECD, 2021).

¿Qué cambios se realizaron en las diferentes asignaturas? ¿En qué se basó el profesorado para realizarlos? ¿Qué significó a nivel personal en el contexto de confinamiento realizar una migración forzada de clases presenciales a un formato $100 \%$ online? Y respecto a las particularidades de la disciplina musical, ¿han sido las clases efectivas en este nuevo formato? ¿Pueden adquirirse las diferentes competencias requeridas en la música de manera online? ¿Qué elementos de las nuevas tecnologías son efectivos con contextos pedagógico-musicales? Las estadísticas oficiales no pueden responder estas preguntas.

De esta manera, surgen los siguientes objetivos de investigación:

- Objetivo General: describir desde el punto de vista del profesorado de Música la experiencia de realizar una readecuación forzada de sus asignaturas a formato online.

- Objetivo Específico 1: describir las modificaciones realizadas por el profesorado.

- Objetivo Específico 2: identificar las diferentes dificultades a nivel profesional y personal que debieron enfrentar los docentes durante este proceso.

- $\quad$ Objetivo Específico 3: analizar las percepciones del profesorado respecto a la efectividad y proyección de la enseñanza online. 


\section{Método}

La investigación se enmarcó en el paradigma constructivista, donde la motivación primordial es "entender y reconstituir las construcciones que las personas sostienen inicialmente, con el objeto de obtener consenso, pero aún estar abiertos a nuevas interpretaciones al ir mejorando la información y la sofisticación" (Guba y Lincoln, 2002, p.143). El enfoque fue cualitativo, dado que la investigación se dio en un entorno natural, donde los investigadores recolectaron e interpretaron los datos obtenidos de diversas fuentes de manera inductiva, mediante un diseño emergente que buscaba describir las perspectivas de los participantes (Creswell y Creswell, 2018). Se utilizó un diseño de investigación compuesto por la superposición del estudio de caso (Yin, 2018) y la teoría fundamentada con comparación constante de Glasser y Strauss (2006).

El estudio de caso se eligió por tratarse de un fenómeno actual, con número definido de participantes situados en un ambiente natural y con múltiples instrumentos de toma de datos. También, se consideró los objetivos de la investigación relacionados con entender las perspectivas de los participantes en contextos naturales (Yin, 2018). La teoría fundamentada fue utilizada en las estrategias de toma y análisis de datos con el fin de generar categorías y teorías ${ }^{1}$ a partir de los datos obtenidos. Tal y como recomiendan Glasser y Strauss (2006) y Strauss y Corbin (2015), no se tomó en cuenta la literatura y teoría existentes en etapas previas al análisis de datos, para evitar contaminar las categorías emergentes con conceptos preestablecidos. A partir de los datos obtenidos a través de múltiples instrumentos que se describen más adelante, se trianguló la información y se establecieron relaciones con la literatura y teoría disponible en la discusión de los resultados.

\subsection{Muestra y entorno}

La muestra contempló a 12 docentes del área de la especialidad de Música de la carrera de Pedagogía en Artes Musicales de una universidad privada ubicada en Santiago de Chile. De acuerdo con el diseño de teoría fundamentada, se utilizó un muestreo teórico intencionado (Glasser y Strauss, 2006).

La carrera de Pedagogía en Artes Musicales se creó el año 2001. En la actualidad, tiene 174 estudiantes y 21 docentes de la especialidad musical. Su objetivo es formar profesorado de Música que se pueda desempeñar en el sistema escolar y otros espacios de desarrollo musical. La estructura curricular contempla ocho líneas formativas musicales: 1) instrumental y vocal; 2)

\footnotetext{
${ }^{1}$ Entendemos teoría, tal como proponen Glasser y Strauss (2006), como relaciones entre las categorías obtenidas.

@ Pedro Iglesias Calonge y Lorena Rivera Pino. The content of this article is the sole responsibility of the authors. The Revista Electrónica de LEEME and Universitat de València are not liable for any legal actions that may arise involving the article's content. Revista Electrónica de LEEME - Lista Electrónica Europea de Música en la Educaciónhttp://ojs.uv.es/index/php/LEEME/index ISSN: 1575-9563. Editores: Universidad de Valencia y Jesús Tejada. Visibilidad de esta revista: SCOPUS, Emerging Sources Citation Index (Clarivate), EBSCO, CINDOC (CSIC), Citefactor, COPAC, Dialnet, DICE (CSIC), DOAJ, e-revistas (CSIC), EBSCO Premier, ERIH+, Gale Cengage Learning, IN-RECS, IRESIE, LATINDEX, MIAR, OCLC Worldcat, RESH, REDIB, RILM Core Journals, SUDOC, ULRICHS. Esta revista es de acceso libre mediante licencia Creative Commons 4.0 CC by. Política de archivado: etiqueta verde SHERPA-ROMEO.
} 
lenguaje musical; 3) cultura musical; 4) ensambles; 5) didáctica; 6) prácticas; 7) tecnología; 8) formación pedagógica.

El perfil del profesorado es, en su mayoría, Profesor/a de Música con Licenciatura en Educación, con diferentes especializaciones y con grado de Magíster. Existen también, en menor número, docentes con Licenciatura en Música, especialmente en las asignaturas instrumentales, y docentes con grado de Doctor para las del área de investigación.

\subsection{Instrumentos e intervenciones}

Para la recogida de datos, se organizó un encuentro de docentes que se denominó "Jornada de intercambio de experiencias virtuales en música", que se realizó en la plataforma Zoom en diciembre del 2020. Se invitó a participar de manera voluntaria a todo el profesorado de especialidad. Los objetivos de la jornada fueron: 1) compartir a nivel de comunidad educativa los procesos de aprendizaje realizados en la readecuación de asignaturas en el contexto online, 2) evaluar los resultados de aprendizaje de los contenidos originalmente planificados para cada curso en el programa; y 3) reflexionar sobre la proyección de la enseñanza online y su integración a actividades pedagógicas en contextos presenciales. Se le pidió a cada docente preparar una breve exposición de no más de diez minutos donde: 1) describieran las principales metodologías utilizadas en la readecuación de clases en formato online; 2) reflexionaran sobre los resultados de las metodologías utilizadas; 3) informaran las principales dificultades que tuvieron; y 4) compararan con metodologías presenciales.

Además, se realizaron dos focus group con el fin de discutir y reflexionar sobre la proyección de la enseñanza online y su integración en actividades pedagógicas en contextos presenciales, que podría producirse una vez que las clases pudieran volver a la normalidad. Los focus group son instancias donde se puede identificar problemas y evaluar procesos de manera efectiva (Morgan y Krueger, 1998). Las preguntas apuntaban a la discusión y evaluación de diferentes metodologías y didácticas aplicadas en clases en formato online para poder explorar sus debilidades y fortalezas. Las sesiones duraron aproximadamente una hora y tuvieron seis participantes cada una.

Junto con esos datos, se hizo análisis de documentos oficiales, en particular del Documento de Priorización Curricular. Este documento, que fue solicitado a cada docente por la dirección del programa, da cuenta de manera oficial de las modificaciones de contenidos, didácticas y metodologías que cada docente realizó en su asignatura durante el período 2020.

@Pedro Iglesias Calonge y Lorena Rivera Pino. The content of this article is the sole responsibility of the authors. The Revista Electrónica de LEEME and Universitat de València are not liable for any legal actions that may arise involving the article's content. Revista Electrónica de LEEME - Lista Electrónica Europea de Música en la Educación-. http://ojs.uv.es/index/php/LEEME/index ISSN: 1575-9563. Editores: Universidad de Valencia y Jesús Tejada. Visibilidad de esta revista: SCOPUS, Emerging Sources Citation Index (Clarivate), EBSCO, CINDOC (CSIC), Citefactor, COPAC, Dialnet, DICE (CSIC), DOAJ, e-revistas (CSIC), EBSCO Premier, ERIH+, Gale Cengage Learning, IN-RECS, IRESIE, LATINDEX, MIAR, OCLC Worldcat, RESH, REDIB, RILM Core Journals, SUDOC, ULRICHS. Esta revista es de acceso libre mediante licencia Creative Commons 4.0 CC by. Política de archivado: etiqueta verde SHERPA-ROMEO. 


\subsection{Plan de análisis}

Los datos de la jornada y focus group fueron grabados, para luego ser transcritos. Para la codificación se utilizó el software Atlas.ti versión 8. Siguiendo el diseño de teoría fundamentada (Glasser y Strauss, 2006; Hernández et al., 2014; Strauss y Corbin, 2015), en primera instancia, se realizó una codificación abierta, para pasar a la codificación axial, donde se organizan las categorías en niveles de abstracción superiores en torno a ejes temáticos. Una vez que se establecieron categorías principales en la codificación axial, se relacionaron los datos obtenidos con la literatura académica, a través del método de comparación constante. Finalmente, se realizó la codificación selectiva para establecer relaciones y jerarquías entre las categorías obtenidas, buscando la saturación teórica, momento en que los datos obtenidos se tornan repetitivos y no aportan nada nuevo (Bonilla y López, 2016).

\subsection{Consideraciones éticas}

Se puso especial énfasis en el carácter voluntario de las actividades realizadas para la toma de datos. Tanto en la invitación como al comienzo de cada actividad, se aclaró a los/as participantes los objetivos de la investigación y la manera en que serían utilizados los datos. Se garantizó el anonimato de cada participante mediante la codificación de nombres propios en las transcripciones. En esta línea, en la presentación de datos solo se especifica el área de trabajo de cada docente. Cada participante firmó un documento de consentimiento informado que explicitaba los objetivos y el manejo de los datos, garantizando el anonimato.

\section{Resultados y discusión}

La necesidad que surge a raíz de la emergencia sanitaria obliga al profesorado a abandonar de manera forzada y repentina el formato de clases presenciales con el que estaban trabajando. Eso significó que cada docente debió hacer modificaciones metodológicas y curriculares en sus asignaturas. Al respecto, es necesario ser conscientes de que durante todo el año 2020 se vivieron varios momentos que hacen complejo un análisis global, pues las directrices que venían de la autoridad se fueron modificando. Durante el primer semestre de 2020 (marzojulio), estaba la idea de que las clases presenciales se reanudarían en un par de meses, por lo que durante ese período se tuvo como alternativa comenzar con los contenidos teóricos, que en ese momento parecían más abordables en formato online, para luego pasar a contenidos prácticos una vez que se retomaran las clases de manera presencial. Esta primera parte estuvo marcada también por una fuerte resistencia del estudiantado a todo tipo modificaciones y readecuaciones curriculares, que significó la imposibilidad de comenzar varias asignaturas. Otro momento lo podemos identificar a comienzos del segundo semestre 2020 (agosto-diciembre), cuando la idea 
de volver a la presencialidad estaba lejana y dirección, docentes y estudiantes asumen que la única manera de planificar el año es de manera online. Los datos que se presentan a continuación son representativos de ese período, pues se recolectaron al momento de finalizar el segundo semestre, en diciembre de 2020. Para la presentación de los datos, se especifica el área de especialidad de cada docente.

\subsection{Modificaciones de contenidos y metodologías}

De lo manifestado en los "Documentos de Priorización Curricular", es importante mencionar que la totalidad del profesorado declaró que su asignatura se podía realizar online. Algunos identificaron algunos contenidos específicos del plan de estudio que serían difíciles de lograr. No obstante, la gran mayoría declaró que podría abordar los contenidos planificados. Respecto a los cambios metodológicos, la respuesta es variada. Algunos consideran que no tuvieron que hacer grandes cambios:

"Principalmente en estas asignaturas [área instrumental], las clases han sido sincrónicas y han sido mayoritariamente como es una clase presencial. La metodología, bueno principalmente es trabajo práctico, no ha existido una gran diferencia en lo que es una clase presencial [...] la respuesta en este caso de la plataforma Zoom ha sido bastante buena, la simultaneidad ha caminado bien (docente área instrumental)".

En otras palabras, el profesorado realiza lo mismo que hacía en el formato tradicional, pero ahora por videoconferencia. Hay que considerar que las clases de instrumento principal son individuales, por lo que la latencia en la transmisión no es un problema. En asignaturas instrumentales grupales, se han tenido que realizar transformaciones convirtiéndolas en clases individuales:

"La clase en realidad se ha transformado casi en una clase individual, porque no pueden trabajar todos al mismo tiempo por lo virtual (docente área instrumental)".

Docentes de otras áreas también manifiestan:

"Bueno yo he adaptado el formato que tenía en la clase presencial. Lo usé en el formato online usando la misma metodología siempre, pero cambié un poco el orden de los contenidos, la manera de profundizarlo (docente lenguaje musical)".

En este caso, el docente manifiesta modificaciones en el orden de los contenidos, sin hacer cambios metodológicos.

"En este sentido, más que una metodología, ha sido como una forma de exponer los contenidos, porque, en el fondo, para la clase de instrumento ha sido prácticamente igual (docente área instrumental)". 
Es decir, parte del profesorado siguió utilizando la misma metodología que en sus clases en formato presencial, pero trasladándola al formato online. Sin embargo, otros creen que es necesario una readecuación metodológica:

\footnotetext{
"No se debe pensar justamente en plantear un periodo online haciendo un símil a lo presencial. Como que no llega a equipararse nunca. Por lo menos en esta carrera, requiere un monitoreo presencial (docente área didáctica)".
}

Al respecto, un docente del área de conjuntos instrumentales manifiesta haber hecho varias modificaciones, tanto de contenidos como metodológicas:

\begin{abstract}
"Este primer semestre yo lo planteé desde un punto de vista más teórico, pensando en la posibilidad de que ellos tuvieran la visión de poder plantear distintos talleres y una propuesta a ofrecer en el mercado laboral. O sea, tener en su mente un taller que ellos quisieran realizar, pero además tener claramente cuál es el costo de ese taller, cuáles son los objetivos de ese taller (docente área ensambles)".
\end{abstract}

Es decir, el profesorado, ante la imposibilidad de realizar su clase de agrupaciones instrumentales, optó por ver contenidos relacionados con la temática de su asignatura, como planificación de talleres orientados al mercado laboral. Esto es considerado por el profesorado como un punto de vista "más teórico", aunque a nuestro modo de ver es una modificación que apunta hacia el Aprendizaje Basado en Proyectos (ABP), con un enfoque hacia el diseño de productos que puedan ofrecer en un mercado laboral, más que un punto de vista teórico. El ABP es una metodología de aprendizaje que se viene aplicando con éxito desde 1960 en educación superior (Zimmermann, 2018). Busca desarrollar "conocimientos y habilidades del siglo XXI trabajando a partir de proyectos en donde [los estudiantes] investigan en torno a un problema, desafío o pregunta compleja que sea auténtica y motivadora" (MINEDUC, s.f). Lo observado en este tipo de metodologías es que el estudiantado se involucra de forma activa en su aprendizaje, tomando la iniciativa para resolver problemas y donde el rol del profesorado es de mediador del proceso de aprendizaje más que un transmisor del conocimiento. Estas características hacen del ABP una metodología que puede ser útil en contextos de enseñanza online, al fomentar la autonomía del estudiantado, "sin tener que depender constantemente del profesor" (Manzanares, 2018, p.18).

Si bien estas estrategias se vienen aplicando con éxito desde hace muchos años, en el contexto de pandemia, hay documentación de aplicaciones en educación exitosas, logrando que: 1) mejore la interacción profesor-alumno; 2) aumente la autonomía del estudiantado; 3 ) mejore el sistema de evaluación, evitando problemas de autoría; y 4) se diversifiquen las herramientas tecnológicas (Llorens-Largo et al., 2021).

El aumento en la motivación de los participantes es una de las grandes ventajas del ABP (Larmer et al., 2015). Esto pudo ser observado por los docentes que lo utilizaron: 
"Hubo un cambio bastante fuerte en la participación de los estudiantes, [...] en ese sentido hubo un cambio muy fuerte (docente lenguaje musical)".

Otros docentes hicieron modificaciones que apuntan al ABP:

\begin{abstract}
"También [cambié] el tipo de evaluación. Antes era la típica prueba de desarrollo, yo les hacía preguntas y ellos me respondían. Ahora lo he orientado más a productos. Les decía: "usted tiene que escribir un artículo de un libro con tal tema" y les daba las directrices (docente área cultura)".
\end{abstract}

Desde hace algunos años, se viene promoviendo en Educación Superior el cambio del esquema de enseñanza tradicional, donde el profesorado es un transmisor del conocimiento, representado por metodologías de clase expositiva o lección magistral, por esquemas centrados en el estudiantado (De Miguel, 2006). Estas nuevas metodologías, que reciben el nombre de "Metodologías Activas", incluyen entre otras al Aprendizaje Basado en Problemas o Proyectos, Aula Invertida, Trabajo Cooperativo y Contrato de Aprendizaje (Jiménez, Gonzáles y Tornel, 2020). Lo observado en tiempos de pandemia es que este tipo de metodologías se habría adaptado bien a las modificaciones del contexto online, mejorando la comunicación entre discentes y docentes y flexibilizando los esquemas tradicionales de clases (Gómez-Hurtado et al., 2020).

Otra variante metodológica perteneciente a las metodologías activas apareció en asignaturas instrumentales:

"Se trabajará con la metodología de Aula Invertida [...] Los estudiantes verán un vídeo explicativo de la materia junto a otros recursos de apoyo disponibles en Campus Virtual. Sobre cada contenido de estudio se practicará en forma autónoma durante la semana, antes de la clase sincrónica (Documento de Priorización Asignatura Área Instrumental)”.

El Aula Invertida es un método pedagógico basado en teorías constructivistas que emplea estrategias de presentación de contenidos de manera asincrónica, habitualmente en forma de vídeos, en momentos previos a la clase. De esta manera, la clase es utilizada para realizar tareas activas de resolución de problemas (Bishop y Verleger, 2013). Es importante hacer notar que Aula Invertida no es simplemente la presentación de contenidos en formato vídeo. No se trata solamente de que el estudiante vea el vídeo, sino que llegue a la clase con los contenidos asimilados, con el fin de utilizar el tiempo de reunión para realizar actividades cognitivas de mayor complejidad. De esta manera, en el momento previo de la clase es cuando se realizan actividades cognitivas de nivel inferior (recordar, entender) y en el momento de la clase, guiados por el profesor, se pueden realizar actividades relacionadas con los niveles superiores (aplicar, analizar, evaluar y crear) (Tucker, 2012).

En disciplinas relacionadas con la música, esta metodología ha sido utilizada con efectos positivos, aumentando la motivación y fomentando actividades cognitivas de orden superior (Doi, 2016; Ma, 2021). 


\subsection{La incorporación de vídeos como recurso de aprendizaje}

Aunque no necesariamente en el contexto de la metodología de Aula Invertida, la mayoría del profesorado utilizó vídeos en sus asignaturas:

"[Utilicé vídeos], tanto como trabajo de un proceso o como parte de una evaluación. Yo antes lo hacía con bastante discreción, porque me sentía incapaz de exigirlo, puesto que yo no sabía si los alumnos tenían o no tenían los medios suficientes para hacerlo (docente área cultura)”.

No solo el profesorado realizó vídeos, sino también el alumnado para diferentes actividades formativas y evaluativas. Mediante los vídeos, también se ha podido abordar de alguna manera la ejecución grupal, que de manera sincrónica presenta serias dificultades por problemas de latencia en la transmisión. En este caso, han tenido que grabar y editar audio para el trabajo de ensamble:

"[Los estudiantes] van grabando la primera voz, luego graban la segunda voz, tercera voz y cuarta voz, con posibilidad de edición. Por lo tanto, aquí ellos aprenden a ocupar y a realizar un trabajo de edición musical en lo que es una sección de bronces (docente área instrumental)".

Respecto a la utilización del vídeo en diferentes instancias de la clase, nuestra toma de datos alcanzó saturación teórica.

"El tema vídeos es como una nueva dimensión que se abrió ahora y creo que también va a pasar a ser parte de las competencias que debe tener un buen profesor. Hacer un buen vídeo, así como una buena dicción, una buena expresión oral, va a ser un algo que se va a requerir del profesor (docente área cultura)".

Otro aspecto que puede potenciar el vídeo es la motivación del alumnado al utilizarlo en instancias de evaluación y retroalimentación.

\footnotetext{
"Bastante sorprendente es el resultado de los vídeos. [...] El vídeo hace que el alumno, por querer alcanzar su mejor versión, tenga que trabajar, y eso es muy bueno, es muy bueno (docente área instrumental)".
}

Junto con posibilitar la evaluación, el estudiantado realiza un proceso autoevaluativo que los motiva en su aprendizaje:

"Los estudiantes graban su mejor versión, envían su mejor versión y les sirve eso también para tener para hacer un estudio más metódico y preocuparse más en lo que están de su aprendizaje (docente área instrumental)".

Varios docentes coinciden con esta apreciación, destacando que la realización de vídeos para efectos evaluativos ha significado que aumente su capacidad autocrítica. Al respecto, se alcanzó saturación teórica. No obstante, varios docentes señalan que podría haber algunos aspectos importantes a considerar. Algunos tienen que ver con el control de situaciones de pánico escénico que podrían ser observadas en una evaluación normal. Sin embargo, esto no es visto 
como una debilidad, sino que nos permitiría observar todo el potencial artístico de un estudiante sin que su rendimiento baje por situaciones de estrés o nervios.

"El vídeo, de alguna otra forma, anula el factor nervios y podemos evaluar objetivamente las habilidades musicales e interpretativa del estudiante (docente área instrumental)".

Además de permitir la posibilidad de editarlo y producir una distorsión de la realidad:

"A veces el vídeo es muy maquillado, o sea, muestra una realidad ficticia que no es necesariamente lo que es la lectura real (docente área lenguaje)".

La incorporación de vídeos como recurso de aprendizaje no es algo nuevo en educación. Los primeros intentos de utilizarlo como recurso pedagógico se remontan a los inicios de la televisión en la década de los 60. En ese entonces, se difundió el "Cono de la Experiencia" de Dale (1964), que ponía la enseñanza a través de medios audiovisuales por encima las metodologías verbales tradicionales. En la actualidad, se piensa que el aprendizaje se ve potenciado a través de los medios multimedia, debido a que la información ingresa al cerebro por múltiples canales (visual y auditivo) (Mayer, 2009). Esto estaría alineado con teorías propuestas desde las neurociencias, que indican qué metodologías multisensoriales podrían en determinadas condiciones ser más efectivas (Salas, 2008).

Sin embargo, es importante subrayar que la inclusión de vídeos, o cualquier innovación tecnológica, no tendrá buenos resultados si no se planifican y alinean con un contexto pedagógico adecuado. Un diseño centrado en la tecnología, hasta la fecha, siempre ha derivado en implementaciones y promesas, para luego decepcionar (Mayer, 2009). El enfoque adecuado sería el centrado en el estudiante, que tiene en cuenta cómo funcionan los mecanismos de cognición humana y adaptan la tecnología para ser aplicada en ese marco.

\subsection{Nueva jornada de trabajo en formato online}

El profesorado siente que todos estos cambios han aumentado su carga laboral.

“[...] para nosotros como profesores sólo aumenta nuestra carga de trabajo, $[\ldots]$ no hemos hablado todavía del gran desgaste que tenemos nosotros como docentes en cuanto a nuestra carga y a trabajar todo un año en una zona que no es nuestro confort (docente área de didáctica)".

Sobre ese tema se alcanzó la saturación teórica, prácticamente todos sienten que el trabajo ha aumentado considerablemente: "Trabajo el triple, $[\ldots]$ porque estoy el día lunes desde las 8 de la mañana hasta las 6:20,
esperando que los estudiantes lleguen a clases (docente área instrumental)".

\footnotetext{
@ Pedro Iglesias Calonge y Lorena Rivera Pino. The content of this article is the sole responsibility of the authors. The Revista Electrónica de LEEME and Universitat de València are not liable for any legal actions that may arise involving the article's content. Revista Electrónica de LEEME - Lista Electrónica Europea de Música en la Educación-. http://ojs.uv.es/index/php/LEEME/index ISSN: 1575-9563. Editores: Universidad de Valencia y Jesús Tejada. Visibilidad de esta revista: SCOPUS, Emerging Sources Citation Index (Clarivate), EBSCO, CINDOC (CSIC), Citefactor, COPAC, Dialnet, DICE (CSIC), DOAJ, e-revistas (CSIC), EBSCO Premier, ERIH+, Gale Cengage Learning, IN-RECS, IRESIE, LATINDEX, MIAR, OCLC Worldcat, RESH, REDIB, RILM Core Journals, SUDOC, ULRICHS. Esta revista es de acceso libre mediante licencia Creative Commons 4.0 CC by. Política de archivado: etiqueta verde SHERPA-ROMEO.
} 
Otro punto importante es que el trabajo frente a la pantalla parece agotador:

"Porque justamente el cambio más grande que yo tengo es que parto a las 8 de la mañana y apago el computador a las 11 de la noche (docente área cultura)".

El trabajo desde el hogar además perturbaría el ambiente familiar:

"En todo este tiempo de desgaste de ambiente familiar. Y uno está sentado en el computador, es super agotador (docente área instrumental)".

Junto con la situación de trabajo desde casa, hay que sumar que gran parte del material que el profesorado utilizaba en sus clases durante años no es compatible con el nuevo formato online, por lo que han tenido que rehacer o modificar su material de trabajo:

"Prácticamente nada de lo que se utilizaba en la clase presencial se puede utilizar acá (docente área lenguaje musical)".

Sobre todo, el agotamiento proviene de la situación de realizar clases en un ambiente que les es ajeno:

"Estamos todos como agotados. Es que este formato conlleva mucho más trabajo. Pero creo que lo más difícil, más que el cansancio, ha sido cómo mantener a los estudiantes motivados, [...] esto para mí ha sido difícil, porque mantenerme enérgica, motivadora, cuando una está tan cansada... (docente área lenguaje musical)".

Si bien la pedagogía es una profesión agotadora y demandante, el profesorado está acostumbrado a recibir retroalimentación de sus estudiantes, siendo una fuente de energía que lo motiva en el ejercicio de su labor diaria.

"Pero en esta versión online donde uno está frente a la computadora en realidad como que falta más retroalimentación, como energía, no sé cómo decirlo, pero hay una cosa emocional que tiene que ver con el contacto físico. [...] a mí me cuesta tener la misma energía que tengo en formato presencial (docente área lenguaje musical)".

\subsection{El futuro del formato online}

Pese a la sobrecarga de trabajo y situaciones de desmotivación, el profesorado considera estos cambios como algo positivo, visualizando las ventajas que aparecen en formatos no presenciales:

"La verdad que se abrió un mundo amplio para todos nosotros, [...], en ese sentido yo creo que va a ser de mucha utilidad (docente área prácticas)".

Esto haría que la forma de impartir clases cambie:

"Yo creo que, de cierta forma, las clases van a cambiar, [...] todo el trabajo que hemos hecho, crear material y todo ese material va a quedar y lo vamos a poder usar perfectamente (docente área vocal)".

@Pedro Iglesias Calonge y Lorena Rivera Pino. The content of this article is the sole responsibility of the authors. The Revista Electrónica de LEEME and Universitat de València are not liable for any legal actions that may arise involving the article's content. Revista Electrónica de LEEME - Lista Electrónica Europea de Música en la Educación-. http://ojs.uv.es/index/php/LEEME/index ISSN: 1575-9563. Editores: Universidad de Valencia y Jesús Tejada. Visibilidad de esta revista: SCOPUS, Emerging Sources Citation Index (Clarivate), EBSCO, CINDOC (CSIC), Citefactor, COPAC, Dialnet, DICE (CSIC), DOAJ, e-revistas (CSIC), EBSCO Premier, ERIH+, Gale Cengage Learning, IN-RECS, IRESIE, LATINDEX, MIAR, OCLC Worldcat, RESH, REDIB, RILM Core Journals, SUDOC, ULRICHS. Esta revista es de acceso libre mediante licencia Creative Commons 4.0 CC by. Política de archivado: etiqueta verde SHERPA-ROMEO. 
Si bien ha sido un esfuerzo importante el aprendizaje de nuevas tareas, el profesorado considera que en el futuro seguirá utilizando estas nuevas herramientas. Además, se percibe la sobrecarga laboral como algo temporal:

"Yo también estoy con la misma sensación de ustedes, de que estoy trabajando el triple, pero lo bueno de esto [...], los tutoriales, van a quedar grabados. Entonces el próximo año no voy a tener que hacer todos los tutoriales de nuevo (docente área cultura)".

Cuando se pregunta si creen que en el futuro la modalidad online podría reemplazar a la presencial, hay consenso absoluto: de ninguna manera. Una modalidad mixta es la que ven como más factible. Algunas razones para este argumento:

"Los aprendizajes de la parte de ellos fueron mucho más lentos que cuando se hace presencial (docente área lenguaje)".

Si bien se podría argumentar que el aprendizaje fue lento debido a la poca experiencia que el profesorado tenía en contextos de enseñanza online, aparecen razones que tienen que ver con aspectos motivacionales que se fomentan en una clase presencial:

"Hay ciertas energías, cosas que se conectan cuando los cabros están trabajando en grupo. Y eso se perdería por mucha tecnología que tuviéramos (docente área instrumental)".

Relacionadas también con el área musical:

"Yo apunto a lo mixto. No se compara el poder tocar en vivo. Para un músico el poder tocar en vivo es como para un jugador de fútbol cuando entra a la cancha, la energía, las emociones y por último el reconocimiento del público, el aplauso (docente área instrumental)".

Y, también, con el área pedagógica, que tiene que ver con el contacto humano necesario para realizar la docencia:

"El tema grave, es que la cosa pedagógica es terrible. Estamos a lo mejor pensando en que, yo lo decía el otro día en una conferencia, solo el ser humano enseña humanidad, por lo tanto, es necesario el tema y el contacto (docente área prácticas)".

En el momento de realizar una evaluación general del período, se argumenta que se requerirá algún tiempo para sacar conclusiones:

"Imposible realizar conclusiones a esta altura del proceso, solo podemos precisar que estamos atentos a los cambios diarios el estado anímico de los protagonistas. [...] solo después que esto termine será posible analizar lo sucedido y realizar una comparación con años anteriores para identificar resultados y cualquier avance que pretendamos hacer en relación con logros. En este momento cabe dentro de la especulación, por ahora decir que hemos velado por el mejor cumplimiento de los resultados de aprendizaje declarados (docente área prácticas)”.

Es importante considerar que la integración de tecnologías en educación no es algo nuevo, con intentos de implementación desde hace años a nivel global. En países angloparlantes, existen desde hace tiempo programas académicos en el área de la música que se ofrecen en modalidad

@ Pedro Iglesias Calonge y Lorena Rivera Pino. The content of this article is the sole responsibility of the authors. The Revista Electrónica de LEEME and Universitat de València are not liable for any legal actions that may arise involving the article's content. Revista Electrónica de LEEME - Lista Electrónica Europea de Música en la Educación-. http://ojs.uv.es/index/php/LEEME/index ISSN: 1575-9563. Editores: Universidad de Valencia y Jesús Tejada. Visibilidad de esta revista: SCOPUS, Emerging Sources Citation Index (Clarivate), EBSCO, CINDOC (CSIC), Citefactor, COPAC, Dialnet, DICE (CSIC), DOAJ, e-revistas (CSIC), EBSCO Premier, ERIH+, Gale Cengage Learning, IN-RECS, IRESIE, LATINDEX, MIAR, OCLC Worldcat, RESH, REDIB, RILM Core Journals, SUDOC, ULRICHS. Esta revista es de acceso libre mediante licencia Creative Commons 4.0 CC by. Política de archivado: etiqueta verde SHERPA-ROMEO. 
online, como en Boston University, Indiana University, University of Newcastle, Massachusetts Institute of Technology, Berklee College of Music, entre muchas otras (Henderson, 2007). No obstante, para una implementación exitosa, es necesario un cambio de rol del docente y del estudiante, además de un replanteamiento de las formas de comunicación existentes en la enseñanza presencial (Henderson, 2007). El formato online requeriría una ruptura del modelo de docente como transmisor del conocimiento para transformarse en un formulador de problemas. También requería un alumno con una actitud activa y no un receptor pasivo de información (Henderson, 2017). Esto parece consistente con la incorporación de metodologías activas que vimos en las modificaciones reportadas por los docentes participantes de nuestro estudio.

Como ejemplo más concreto encontramos la investigación de Souza (2017), que reporta los resultados de un estudio experimental de la implementación de un MOOC (Massive Open Online Course) en el área musical. Este curso abordaba las áreas de composición, lenguaje musical, apreciación musical, técnica e interpretación. El estudio concluye que las aplicaciones utilizadas, que fueron implementadas en formato online, "son recursos eficientes para el aprendizaje musical" (Souza, 2017, p.144). También Quílez y Alsina (2021) reportan buenos resultados en cursos online para formación de profesorado en contextos online.

En contraste, la OECD en un estudio a gran escala que relacionaba resultados de la prueba PISA y enseñanza con TIC, no sólo cuestionó su valor educativo, sino que además las responsabiliza del bajo logro alcanzado en las pruebas PISA en algunos países como Emiratos Árabes, Chile, Brasil y Colombia (Castellanos, 2015; OECD, 2015). Si bien es posible atribuir esos resultados a una mala implementación, los mismos estudios concluyen que el aprendizaje requiere interacciones profundas entre el profesorado y el alumnado, que son difíciles en contextos online (OECD, 2015).

A modo de síntesis, se muestra un mapa conceptual con las categorías principales que emergieron de nuestros datos (Figura 1).

@Pedro Iglesias Calonge y Lorena Rivera Pino. The content of this article is the sole responsibility of the authors. The Revista Electrónica de LEEME and Universitat de València are not liable for any legal actions that may arise involving the article's content. Revista Electrónica de LEEME - Lista Electrónica Europea de Música en la Educación-. http://ojs.uv.es/index/php/LEEME/index ISSN: 1575-9563. Editores: Universidad de Valencia y Jesús Tejada. Visibilidad de esta revista: SCOPUS, Emerging Sources Citation Index (Clarivate), EBSCO, CINDOC (CSIC), Citefactor, COPAC, Dialnet, DICE (CSIC), DOAJ, e-revistas (CSIC), EBSCO Premier, ERIH+, Gale Cengage Learning, IN-RECS, IRESIE, LATINDEX, MIAR, OCLC Worldcat, RESH, REDIB, RILM Core Journals, SUDOC, ULRICHS. Esta revista es de acceso libre mediante licencia Creative Commons $4.0 \mathrm{CC}$ by. Política de archivado: etiqueta verde SHERPA-ROMEO. 

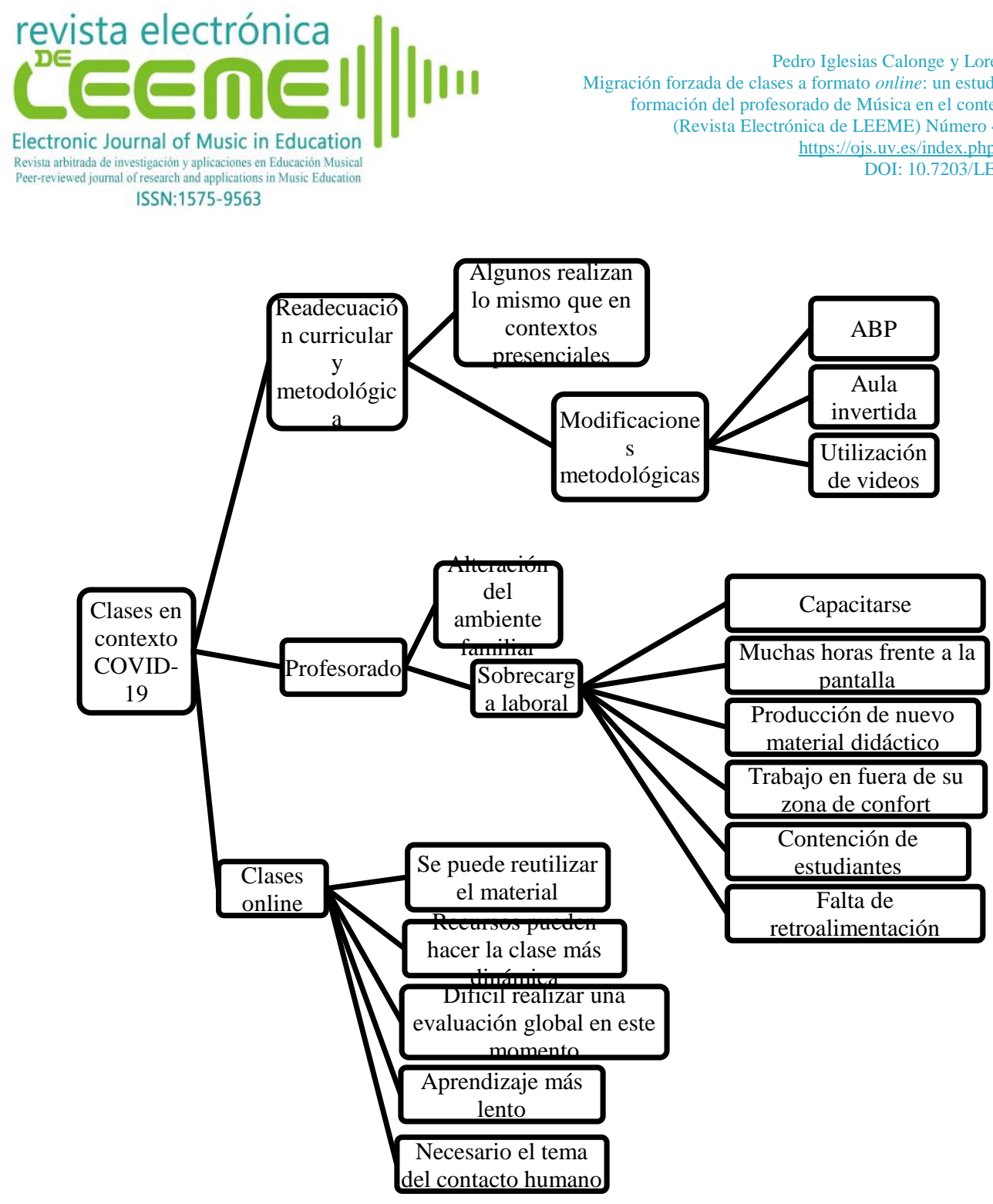

Figura 1. Mapa conceptual de categorías obtenidas

\section{Conclusiones}

Nuestros datos dan cuenta de una gran diversidad de perspectivas por parte del profesorado que participó en el estudio. Las modificaciones en el contexto online, para algunos significaron realizar por videoconferencia lo mismo que realizaban en contextos presenciales. No obstante, la gran mayoría realizó cambios sustanciales en sus asignaturas. Varias de estas modificaciones están relacionadas con las Metodologías Activas, como el Aprendizaje Basado en Proyectos y el Aula Invertida. Junto a eso, el profesorado ve el potencial y las posibilidades que aparecen con la incorporación de vídeos en contextos de aprendizaje.

Por otra parte, el profesorado manifestó haber tenido que soportar una carga laboral importante durante el período de confinamiento, además de alteraciones en su ambiente familiar, 
al tener que realizar su trabajo desde su hogar. Si bien los docentes aprecian algunas de las ventajas y posibilidades que brindan las clases en formato online, la sensación es que los aprendizajes son más lentos que en contextos presenciales, además de dificultarse la comunicación a nivel profundo con el estudiantado, que denominaron "el contacto humano". Este contacto se percibe como un factor motivador importante del aprendizaje.

Después de más de un año funcionando en condiciones de incertidumbre, podría parecer que la enseñanza online está siendo efectiva: las asignaturas han podido finalizar, los estudiantes están aprobando y la matrícula no ha bajado de manera considerable. Se ven en prensa comentarios de "expertos" 2 que hablan de que el futuro de la educación será online. Son varios los problemas que parecen ser solucionados mediante este formato y se ve como algo prometedor. Pero, esta promesa se basa en fundamentos débiles, estamos lejos de poder hacer una evaluación en profundidad sobre el aprendizaje efectivo en este período de emergencia. El profesorado, quien ha sido el responsable de planificar e implementar estas clases, está lejos de tener una visión positiva. Se han revelado muchas deficiencias relacionadas con este tipo de formación y los docentes lo han experimentado en primera línea. Entonces, surge la gran pregunta ¿son las metodologías online más efectivas que las tradicionales? O, dicho de otra manera, ¿saldrán mejores profesionales de nuestras universidades si implementamos programas en este formato? ¿Se puede transmitir información en contextos online? Sí. Muchos estudios dan cuenta de eso. ¿Se puede formar un profesional de la educación en contextos online? La transmisión de información no es lo mismo que la tarea de formar de un profesional. El problema va mucho más allá de superar brechas tecnológicas y solucionar problemas de conectividad. Durante la implementación en 2020, el estudiantado se percibe desmotivado y los docentes están experimentando una sobrecarga laboral importante. ¿Es este el futuro de la educación?

El profesorado ha sido el responsable de implementar sus clases en modalidad online. Para eso, se ha tenido que capacitar y cambiar su modalidad de trabajo en un ambiente que escapa a su zona de confort. Ve que su conocimiento y capacidad profesional se ha enriquecido en ese proceso. La aplicación de Metodologías Activas y la incorporación de vídeos como recurso educativo ha tenido un buen resultado y considera que muchos de estos elementos le servirán al momento de retornar al formato presencial. Esto no es de sorprender, estas metodologías tienen un sólido fundamento pedagógico. Ninguno de los datos obtenidos apunta a que el proceso formativo haya mejorado en formato online. Todo esto se ve por el profesorado como una respuesta a la contingencia, no como un intento sistemático que tenga proyección a largo plazo. No basta con implementar un aula virtual y subir contenidos a la red, no son las máquinas las que enseñan, es el profesorado quien enseña a través de las máquinas. Las TIC son una herramienta más, tal como es el libro, la pizarra o un lápiz. Si queremos que las modalidades de educación

\footnotetext{
${ }^{2}$ Véase por ejemplo "Impacto de la modalidad online. El futuro de la educación superior en Chile" (Hevia, 2021) o "La enseñanza en línea genera desarrollo" (2021).

@ Pedro Iglesias Calonge y Lorena Rivera Pino. The content of this article is the sole responsibility of the authors. The Revista Electrónica de LEEME and Universitat de València are not liable for any legal actions that may arise involving the article's content. Revista Electrónica de LEEME - Lista Electrónica Europea de Música en la Educaciónhttp://ojs.uv.es/index/php/LEEME/index ISSN: 1575-9563. Editores: Universidad de Valencia y Jesús Tejada. Visibilidad de esta revista: SCOPUS, Emerging Sources Citation Index (Clarivate), EBSCO, CINDOC (CSIC), Citefactor, COPAC, Dialnet, DICE (CSIC), DOAJ, e-revistas (CSIC), EBSCO Premier, ERIH+, Gale Cengage Learning, IN-RECS, IRESIE, LATINDEX, MIAR, OCLC Worldcat, RESH, REDIB, RILM Core Journals, SUDOC, ULRICHS. Esta revista es de acceso libre mediante licencia Creative Commons 4.0 CC by. Política de archivado: etiqueta verde SHERPA-ROMEO.
} 
online tengan éxito, necesitamos profesionales con la convicción pedagógica de que pueden funcionar.

\section{Referencias}

Bishop, J. y Verleger, M.A. (2013). The flipped classroom: A survey of the research. En 2013 ASEE Annual Conference \& Exposition (pp.23-1200). https://doi.org/10.18260/1-2-22585

Bonilla-García, M.A. y López-Suárez, A.D. (2016). Ejemplificación del proceso metodológico de la teoría fundamentada. Cinta de Moebio, 57, 305-315. https://doi.org/10.4067/S0717$\underline{554 X 2016000300006}$

Castellanos, M. (2015). ¿Son las TIC realmente, una herramienta valiosa para fomentar la calidad de la educación? UNESCO, Organización de las Naciones Unidas para la Educación, la Ciencia y la Cultura. https://unesdoc.unesco.org/in/rest/annotationSVC/

Creswell, J. y Creswell, D. 2006. Research Design: Qualitative, Quantitative, and Mixed Methods Approaches. SAGE.

Dale, E. (1964). Métodos de Enseñanza Audiovisual. Editorial Reverté.

De Miguel, M. (2006). Metodologías de enseñanza y aprendizaje para el desarrollo de competencias: Orientaciones para el profesorado universitario ante el Espacio Europeo de Educación Superior. Alianza.

Doi, C. (2016). Applying the flipped classroom methodology in a first-year undergraduate music research methods course. Music Reference Services Quarterly, 19(2), 114-135. https://doi.org/10.1080/10588167.2016.1167427

Glaser, B. y Strauss, A. (2006). Discovery of grounded theory: Strategies for qualitative research. Aldine Transaction.

Gómez-Hurtado, I., García-Rodríguez, M.P., González Falcón, I. y Coronel Llamas, J.M. (2020). Adaptación de las Metodologías Activas en la Educación Universitaria en Tiempos de Pandemia. Revista Internacional de Educación para la Justicia Social, 9(3), 415-433. https://doi.org/10.15366/riejs2020.9.3.022

Guba, E. y Lincoln, Y. 2002. Paradigmas en competencia en la investigación cualitativa. En Denman y Haro (Eds.), Por los rincones. Antología de métodos cualitativos en la investigación social (pp.113-145). Región y Sociedad. 
Henderson, J.R. (2007). Formação continuada de professores de música em ambiente de ensino e aprendizagem online. (Tesis Doctoral). Universidade Federal do Rio Grande do Sul. http://hdl.handle.net/10183/11229

Hernández, R., Fernández, C. y Baptista, P. (2014). Metodología de la investigación. Mc Graw Hill.

Hevia, V. (2021). Impacto de la modalidad online: El futuro de la educación superior en Chile. EMOL. https://www.emol.com/noticias/Tendencias/

Jiménez, D., Gonzáles, J. y Tornel, M. (2020). Metodologías activas en la universidad y su relación con los enfoques de enseñanza. Profesorado, Revista de Currículum y $\begin{array}{llll}\text { Formación del Profesorado, } & \text { 24(1), }\end{array}$ https://doi.org/10.30827/profesorado.v24i1.8173

La enseñanza en línea genera desarrollo. (2021). El Comercio. https://www.elcomercio.com/

Larmer, J., Mergendoller, J. y Boss, S. (2015). Setting the standard for project based learning. ASCD.

Llorens-Largo, F., Villagrá-Arnedo, C., Gallego-Durán, F. y Molina-Carmona, R. (2021). COVID-proof: cómo el aprendizaje basado en proyectos ha soportado el confinamiento. Campus Virtuales, 10(1), 73-88. http://uajournals.com/ojs/

Ma, R. (2021). Stimulating students learning motivation: A case study of music education and pedagogy course based on flipped classroom. Psychology and Education Journal, 58(2), 109-114. https://doi.org/10.17762/pae.v58i2.1059

Manzanares, A. (2018). Sobre el aprendizaje basado en problemas. En A. Escribano y A. del Valle. (Eds), El aprendizaje basado en problemas: una propuesta metodológica en educación superior. Narcea Ediciones.

Marinoni, G., Van't Land, H. y Jensen, T. (2020). The impact of Covid-19 on higher education around the world. International Association of Universities. https://www.iau-aiu.net/

Mayer, R. (2009). Multimedia Lerning. Cambridge University Press.

MINEDUC Chile (s.f). ¿Qué es ABP? https://www.curriculumnacional.cl/portal/ABP/

Morgan, D. y Krueger, R. (1998). The Focus Group Guidebook. SAGE.

OECD (2015). Students, Computers and Learning: Making the Connection. OECD. https://doi.org/10.1787/9789264239555-en

@Pedro Iglesias Calonge y Lorena Rivera Pino. The content of this article is the sole responsibility of the authors. The Revista Electrónica de LEEME and Universitat de València are not liable for any legal actions that may arise involving the article's content. Revista Electrónica de LEEME - Lista Electrónica Europea de Música en la Educación-. http://ojs.uv.es/index/php/LEEME/index ISSN: 1575-9563. Editores: Universidad de Valencia y Jesús Tejada. Visibilidad de esta revista: SCOPUS, Emerging Sources Citation Index (Clarivate), EBSCO, CINDOC (CSIC), Citefactor, COPAC, Dialnet, DICE (CSIC), DOAJ, e-revistas (CSIC), EBSCO Premier, ERIH+, Gale Cengage Learning, IN-RECS, IRESIE, LATINDEX, MIAR, OCLC Worldcat, RESH, REDIB, RILM Core Journals, SUDOC, ULRICHS. Esta revista es de acceso libre mediante licencia Creative Commons 4.0 CC by. Política de archivado: etiqueta verde SHERPA-ROMEO. 
OECD (2021). The state of higher education: One year in to the COVID-19 pandemic. OECD. https://doi.org/10.1787/83c41957-en

Quílez, A. y Alsina, M. (2021). Desarrollo profesional y formación docente online. El caso de "Mini-Deportistas", un proyecto de música y movimiento. Revista de Investigación y Docencia Creativa, 10(18), 1-22. https://doi.org/10.30827/Digibug.67828

Salas, R. (2008). Estilos de aprendizaje a la luz de la neurociencia. Cooperativa Editorial Magisterio.

Souza, T. (2017). Laboratório online de música e tecnologia: planejando e implementando um MOOC para o ensino de música online. (Tesis de Maestría). Universidade Federal de Brasília. https://repositorio.unb.br/handle/10482/31796

Strauss, A. y Corbin, J. (2015). Basics of qualitative research techniques. SAGE.

Tucker, B. (2012). The flipped classroom. Education Next, 12(1), 82-83. http://www.msuedtechsandbox.com

UNESCO (2021). COVID-19: reopening and reimagining universities, survey on higher education through the UNESCO National Commissions. UNESCO. https://unesdoc.unesco.org/ark:/48223/pf0000378174

Yin, R.K. (2018). Case study research and applications: Design and methods. SAGE.

Zimmermann, B. (2018). Prólogo. En A. Escribano y Á. del Valle. (Eds), El aprendizaje basado en problemas: una propuesta metodológica en educación superior. Narcea Ediciones.

@Pedro Iglesias Calonge y Lorena Rivera Pino. The content of this article is the sole responsibility of the authors. The Revista Electrónica de LEEME and Universitat de València are not liable for any legal actions that may arise involving the article's content. Revista Electrónica de LEEME - Lista Electrónica Europea de Música en la Educaciónhttp://ojs.uv.es/index/php/LEEME/index ISSN: 1575-9563. Editores: Universidad de Valencia y Jesús Tejada. Visibilidad de esta revista: SCOPUS, Emerging Sources Citation Index (Clarivate), EBSCO, CINDOC (CSIC), Citefactor, COPAC, Dialnet, DICE (CSIC), DOAJ, e-revistas (CSIC), EBSCO Premier, ERIH+, Gale Cengage Learning, IN-RECS, IRESIE, LATINDEX, MIAR, OCLC Worldcat, RESH, REDIB, RILM Core Journals, SUDOC, ULRICHS. Esta revista es de acceso libre mediante licencia Creative Commons 4.0 CC by. Política de archivado: etiqueta verde SHERPA-ROMEO. 